\title{
Inhalt
}

Seite

Literaturverzeichnis $\ldots \ldots \ldots$

\section{Theorle der Flächenmetrlk}

Einleitung

\section{A. Fächenmetrik}

1. Gaußsche Darstellung der Flächen im dreidimensionalen euklidischen Raum. . . . . . . . . . . . . . . 7

2. Zulässige Parameter. Reguläre Parameternetze . . . . . . 13

3. Einführung neuer zulässiger Gaußscher Koordinaten . . . . 17

4. Flächenkurven. Flächentangenten. Tangentenebene . . . . 19

5. Normalenvektor der Fläche. Die metrischen Fundamentalgrößen $E, F, \theta$, und $W$. Punkte mit isotropen Flächennormalen und isotropen Tangentenebenen ............ 21

6. Linienelement und Metrik einer Fläche. Isotrope Flächenkurven 26

7. Metrisch singuläre Flächen $\left(W^{2}=E G-F^{2} \equiv 0\right) . \ldots 33$

8. Invarianzeigenschaften von $E, F, G, W$ und $d s^{2} \ldots \ldots \ldots$. . 41

9. Kugelmetrik. Kugelloxodromen . . . . . . . . . 44

10. Isotrope Linien und isotrope Parameter der Kugel. Riemannsche Zahlenkugel. Stereographische Projektion . . . . . . . 47

11. Eulersche Darstellung der Flächen . . . . . . . . . . . . 52

12. Drehflächen . . . . . . . . . . . . . . 53

13. Schraubflächen ................. 55

14. Stetige Verbiegung der Kettenfläche in die Wendelfläche. Satz von Edmond Bour. . . . . . . . . . . . 56

15. Isometrie und Verbiegung . . . . . . . . . . . . 67 67

16. Metrik der euklidischen Ebene . . . . . . . . . . 70

17. Kegel . . . . . . . . . . . . . . 72

18. Zylinder ................. . . 74

19. Torsen . . . . . . . . . . . . . . 76

20. Regelflächen ............... . . 80

21. Kehlpunkte und Kehllinie einer Regelfläche . . . . . . . . 86

22. Ableitungsgleichungen, Invarianten, natürliche Gleichungen und Mindingsche Biegungen einer Regelfläche ..... . 99

\section{B. Vektoranalysis auf Flachen}

23. Die Differentiatoren $\vartheta(\varphi, \psi)$ von Darboux und $\nabla \varphi$ von Beltrami. Gradient einer Ortsfunktion auf der Fläche ...... 109

24. Divergenz (Quelldichte) eines Vektorfeldes auf der Fläche . . 119

25. Rotation (Wirbeldichte) eines Vektorfeldes auf der Fläche . . 124 
26. Der zweite Beltramische Differentiator $\Delta \varphi$. Beltramische Differentialgleichungen. Harmonische Funktionen . . . . . . . 128

27. Die Formeln von Green. Das Dirichletsche Problem . . . . . . 138

\section{c. Theorie der Abbildung von Fiăchen}

28. Abbildung zwejer Flächen aufeinander. Berührende Affinität. Längentreue, Winkeltreue, Flächentreue. . . . . . . . . 144

29. Dle Hauptverzerrungsrichtungen einer Abbildung. Indikatrizen von Tissot und Study . . . . . . . . . . . . . . . . 148

30. Konforme Abbildung einer reeilen analytischen Fläche auf die Ebene . . . . . . . . . . . . . . . . . . . . 155

31. Konforme Abbildung zweier reeller analytischer Flächen aufeinander... . . . . . . . . . . . . . . . . . 167

32. Beispiele von konformen (winkeltreuen) Abbildungen der Kugel auf die Ebene. (Mercatorkarte. Stereographische Projektion der Kugel und ihre Verallgemeinerung durch Lambert) . . . . . , 170

33. Beispiele von flächentreuen Abbildungen der $\mathbf{K}$ ugel auf die Ebene. (Entwürfe von Archimedes, Sanson, Mollweide, Eckert, Lambert, Albers, Bonne und Stab-Werner), : . . . . . . . 18

\section{Geodätische Krïmmung. Geodātische Lỉn ien. Absoluter Parallelismus}

34. Geodätische Krümmung einer Flächenkurve . . . . . . . . . 202

35. Geodätische Linien . . . . . . . . . . . . . . . . . . . . 209

36. Minimaleigenschaft der geodätischen Linien . . . . . . . . . 216

37. Differentialgleichung der geodätischen Linien . . . . . . . . 219

38. Geodätische Linien auf Drehflächen und auf Liouvilleschen Flächen... . . . . . . . . . . . . . . 222

39. Invariante Darstellung der geodätischen Krümmung . . . . . 234

40. Parallelverschiebung auf einer Fläche. Absoluter Parallelismus von Levi-Civita. . . . . . . . . . . . . . . . . . 236

41. Autoparallelismus . . . . . . . . . . . . . . . . . . . 244

42. Absolute Differentiation längs einer Flächenkurve. Frenetsche Formeln der absoluten Theorie der Flächenkurven . . . . . 247

Namen- und Sachverzeichnis ............... 253 